\title{
Language Mapping in Temporal Lobe Epilepsy in Children: Special Considerations
}

\author{
Sandrine de Ribaupierre, ${ }^{1}$ An Wang, ${ }^{2,3}$ and Susan Hayman-Abello ${ }^{4}$ \\ ${ }^{1}$ Department of Clinical Neurological Sciences, The University of Western Ontario, Victoria Hospital, Commissioners Road, London, \\ ON, Canada N6A 5W9 \\ ${ }^{2}$ Imaging Research Laboratories, Robarts Research Institute, 100 Perth Drive, London, ON, Canada N6A $5 \mathrm{~K} 8$ \\ ${ }^{3}$ Department of Medical Biophysics, The University of Western Ontario, London, ON, Canada N6A 5C1 \\ ${ }^{4}$ Department of Psychology, London Health Sciences Centre, London, ON, Canada N6A 5C2
}

Correspondence should be addressed to Sandrine de Ribaupierre, sandrine.deribaupierre@lhsc.on.ca

Received 29 July 2011; Accepted 6 November 2011

Academic Editor: Seyed M. Mirsattari

Copyright (C) 2012 Sandrine de Ribaupierre et al. This is an open access article distributed under the Creative Commons Attribution License, which permits unrestricted use, distribution, and reproduction in any medium, provided the original work is properly cited.

\begin{abstract}
Temporal lobe epilepsy (TLE) in children is a slightly different entity than TLE in adults not only because of its semiology and pathology but also because of the different approach to surgical treatment. Presurgical investigations for eloquent cortex, especially language, must take these differences into account. Most diagnostic tests were created for adults, and many of the assessment tools need to be adapted for children because they are not just small adults. This paper will highlight the specific challenges and solutions in mapping language in a pediatric population with TLE.
\end{abstract}

\section{Introduction}

In refractory temporal lobe epilepsy (TLE), it is important to be able to determine which hemisphere is dominant and hosts the majority of the language areas. When a patient is evaluated as a potential candidate for resective surgery, language mapping should be able to indicate which hemisphere is dominant and precisely identify where the language areas are situated within the brain.

While the general principle of mapping language for TLE in children might be the same as for adults, many challenges are encountered in the mapping process because children are not small adults but differ from adults in many aspects.

To understand some of the differences between children and adults in TLE features, a brief overview of TLE is provided, focusing on surgical candidates and preoperative investigations. Then, a brief summary of language development and lateralization differences between normal children and children with epilepsy is provided. Finally, various techniques for language assessment are described.

\section{Temporal Lobe Epilepsy in Children}

The semiology of temporal lobe originating seizures is not as well characterized in children compared to adults and is dependent on age. For example, infants have a predominance of behavioral arrests, they also tend to have more prominent convulsive activity than adults, and their seizures appear clinically generalized. In younger patients, the automatisms are first discrete and mostly orofacial, but the complexity of hand automatisms increases with age. After the age of 3 years, tonic or myoclonic spasms decrease, as do other motor phenomena, which might have been reminiscent of frontal lobe seizures, and the overall semiology becomes closer to that observed in adults $[1,2]$.

The etiology of the seizure in children is also different. Mesial temporal sclerosis (MTS) is the most common adult etiology, while in children it is relatively rare. In the pediatric population, when MTS is present, it is often accompanied by a neocortical pathology (dual pathology) [3-6], and curative surgery therefore necessitates a temporal lobectomy instead of a selective amygdalohippocampectomy to maximize the chances of being seizure-free [7]. Other pathologies, such 
as focal malformation of cortical development, tumors (such as gangliogliomas and dysembryoplastic neuroepithelial tumors), are frequent and also necessitate a neocortical resection. Because language is not only affected by interindividual differences but also variably modified by epilepsy, exact language mapping is required before any neocortical resection to minimize postoperative neurological deficit.

Epilepsy surgery has been shown to lead to better cognitive development $[8,9]$ if the epileptogenic zone can be completely resected, but it also carries a higher risk of some language deficit (up to $50 \%$ in a series) [10].

\section{Language and TLE}

Language is progressively acquired over the years, and its development might be affected by seizures themselves, age at the onset of seizures, seizure severity, and the underlying pathology. Surgery also has a different impact depending on the age at which it is performed.

The general population, independent of handedness, has a $10-18 \%$ chance of having a right or bilateral dominant hemisphere (5\% in a right-handed population and $22 \%$ in a left-handed population [11-14]) the majority of them are left handed. By contrast, among the epilepsy population, 77\% have an atypical pattern for language (right or bilateral) as determined by the Wada test or functional MRI (fMRI) [1517]. Language might be displaced to the contralateral hemisphere or be reorganized within the same hemisphere, either a different location in that hemisphere, or compensated with additional areas recruited [14, 15, 18-25].

It has also been shown that more children with epilepsy have an atypical language network than adults with epilepsy; whether these findings are correlated with the age of onset of the seizures is a matter of debate. Some studies have shown a difference in language pattern with early-onset seizures $[17,19,26]$, while other studies have not been able to show that correlation $[16,27-30]$. In addition, the percentage of atypical language seems even higher when the epilepsy is probably symptomatic (formerly known as cryptogenic, i.e.,: no lesion detected on the MRI) [21]. However, even if language could be influenced by seizures and might be shifted to the contralateral side in some patients, it could also remain in the "normal" anatomical location on the left even when the seizures are arising from that area [31], which is important to remember when considering surgery to treat epilepsy.

While it was initially thought that language lateralization was acquired later in life, recent functional magnetic resonance imaging (fMRI) and magnetoencephalography (MEG) studies have shown signs of lateralization in infants, with the left frontal region being implicated in the discrimination of speech sounds, for example, [32-35]. However, there seems to be an increase in lateralization with age as shown by fMRI and MEG studies in normal children [36, 37], which could explain the better potential outcome in language reorganization following surgery before the age of 5-6 years [38-40]. Most studies have demonstrated a difference in lateralization depending on the task. For example, verbgeneration and story-processing tasks demonstrate more changes in lateralization over time than word-picture matching tasks [36, 41-43].

\section{Tools to Map Language}

All of the tools to assess language were developed in adults and then adapted for pediatric populations. While new technologies allow us to move away from invasive techniques, these technologies still carry challenges when applied to children. When considering all these methods, there are general constraints based on the age of the patient, which will be the same for all.

Currently, language assessment is not performed in infants and toddlers, except in specific research settings; therefore, the following will focus on children who have developed enough language skills to communicate and be tested by visual and/or oral questions.

Because all of the techniques require cooperation of the subject, it is important to keep age-specific abilities in mind when developing the tests. The attention span increases with age and is relatively short in younger children. In addition, antiepileptic drugs as well as cognitive delay resulting from epilepsy might affect attention span.

Cognitive psychologists have made us quite aware of the timeline of language development, which should be considered when developing tests of language function. Development of phonological, semantic, grammatical, and pragmatic components of language during childhood influences the design of the studies. To obtain satisfactory results and map the language accurately, it is essential to use tasks that are appropriate for the age of the patient.

In addition, processes occurring during brain development (such as the formation of synaptic contacts and myelination) also affect most of the imaging and mapping techniques and may thus influence the results. Their influence on each technique will be analyzed individually below. The last point to consider when interpreting the results of a patient compared to a study is that many imaging studies have a relatively small number of subjects, and the subject population is heterogeneous. For the same type of study, a pediatric study should have a larger number of subjects than an adult study because there is significantly more variability among children due to development, yielding an even more heterogeneous population.

The various mapping techniques are described below, beginning with neuropsychological assessment, moving to more invasive tests, such as cortical stimulation, sodium amobarbital (Wada) test, and nuclear medicine (SinglePhoton Emission Computed Tomography_SPECT-and Positron Emission Tomography-PET), then to contemporary tools, which are being used increasingly more in the clinic (fMRI and MEG), and finally to newer tools that are currently being assessed, such as functional Nearinfrared spectroscopy (fNIRS) and diffusion tensor imaging (DTI). 
4.1. Neuropsychological Evaluation. In the context of epilepsy, clinical language assessment begins with a neuropsychological assessment that helps to determine lateralization and guides the decision as to whether more in-depth assessment is needed (i.e., if language is thought to be on the left and the surgery is a right temporal lobectomy, it is not generally necessary to investigate further). The neuropsychological assessment includes a battery of standardized, age-appropriate tests for language and memory, among other cognitive domains, such as attention, visual-spatial skills, motor skills, and executive functions, interpreted in the context of developmental milestones, academic skills, psychosocial functioning, and so forth [44]. This global assessment helps determine assets and deficits and detect whether the pattern is consistent with dysfunction in a specific region of the brain or with a known neurological syndrome.

As part of the neuropsychological test battery, fused dichotic word listening tests (FDWLTs) have proven to be cost-effective, noninvasive methods for identifying language dominance as left, right, or bilateral in adults, children, and adolescents [45-47]. During this behavioral test, different words are presented to both ears simultaneously, and the subject reports which one they heard. The number of correct answers for each ear is counted, and the value indicates a right or left ear advantage. The rationale behind this test is that contralateral projections from the ear to the brain are stronger than ipsilateral projections [48].

It is well known that ongoing seizures in refractory epilepsy can affect development therefore by reducing the number of seizures, surgery does help in ameliorating learning and general development deficits. However, studies show that temporal lobectomy of the dominant hemisphere, even in children, leads to some degree of postoperative deficit in language. A study of 24 children with complex partial seizures, aged 5.8-15.7 years, showed a preoperative leftlanguage dominance in $65 \%$ of subjects, with an estimated language delay of 1.7-3.5 years. Postoperatively, these same children had an increase in language delay in all areas except for receptive syntax [49]. This increased language delay postoperatively might be less of a problem in younger children [50].

While language lateralization is important, it is not the only factor when considering surgery. The traditional notion of two areas well demarcated anatomically within the frontal and temporal lobes-Broca and Wernicke-has been replaced by the knowledge of a language network that has some interindividual variability [51]. When further information beyond laterality alone is required, imaging or further investigations with more language specificity are undertaken.

4.2. Sodium Amobarbital or Wada Test. The Wada test consists of an injection of intracarotid sodium amobarbital to freeze half of the brain to lateralize function. The procedure consists of a dose of 40-125 mg (depending on the body weight) of sodium amobarbital into the internal carotid through a femoral catheter. The catheter is usually inserted under general anesthesia. Once the patient has returned to a normal baseline after anesthesia, the side where the seizure focus is present is tested first followed by the opposite side. The contralateral injection is typically performed 30 45 minutes after the first using the same procedure as for the first. Hemiplegia is first examined, and then language is tested either by a pediatric epileptologist or neuropsychologist. This is subject to variation from one centre to the next and is sometimes even performed on different days.

The sodium amobarbital test requires full cooperation of the child, who has been subjected to a stressful situation because the injection also causes transient hemiplegia. While the situation can be explained and tolerated by older children (teenagers) [52, 53] or adults, it is extremely difficult in younger children. Nonetheless, successful sodium amobarbital testing has been reported in children as young as 2 years old $[53,54]$. In a study that tested 22 patients between the ages of 5 and 12 years (median 10), language lateralization was clearly identified in $50 \%$ of patients; ten children had left hemispheric language while one had a right hemispheric dominance. Furthermore, the percentage of successful sodium amobarbital procedures was higher among children with higher IQs (100\% over IQ 70, 57\% of IQ < 70) [53]. A later study from the same group reported a $62.5 \%$ success rate in 42 children; $7.5 \%$ failed because of inconclusive results from the test (intact language after both injections, or a mix of intact language after one injection and then noncooperation), and 30\% failed because of inadequate cooperation [55]. Similarly, Schevon reported a successful sodium amobarbital test in $57 \%$ of patients younger than 10 years but as high as $93 \%$ in patients older than 10 years of age [54].

However, the information obtained during the sodium amobarbital test is whether language is impaired after injecting sodium amobarbital into a particular side and therefore determines the dominant side. It might help to detect some bilateral patterns and predict language deficit after a proposed surgery; however, a cortical map of the language network cannot be created based on this procedure. Another useful function of the sodium amobarbital test, which will not be discussed here, is to assess memory, which can be performed in the same setting.

4.3. SPECT and PET. Nuclear imaging, such as SinglePhoton Emission Computed Tomography (SPECT) and Positron Emission Tomography (PET), has been used in the past to map eloquent cortex, including language areas. However, even though studies have shown good correlation between nuclear imaging and intraoperative language mapping [56] or sodium amobarbital tests [57], such imaging exposes children to radiation. Furthermore, these imaging methods are limited by the lack of spatial and temporal resolution [57-59]. SPECT (especially SISCOM techniques - the subtraction of the ictal and interictal SPECT then registering to the MRI) and PET are still used to find the epileptogenic zone, but, currently, they are not as commonly used to map language $[60,61]$. 
4.4. Cortical Stimulation/Mapping. Cortical stimulation to map eloquent cortex was described by Penfield in the early 1950s [62]. However, because the procedure required a cooperative patient in the operating room, until the advent of implanted subdural or depth electrodes, it was only amenable to older children or adults.

Leaving subdural electrodes in place allows the use of cortical stimulation in the perioperative period. It also allows distribution of the language tests and mapping on different days, which is more suitable to children's shorter attention spans. However, because it is invasive and carries some surgical risks, subdural electrodes, grids, or depth electrodes would not only be implanted for language mapping, but might also be used when invasive recording is otherwise necessary to determinate the epileptogenic zone. Of note, only a restrictive part of the brain is exposed and, therefore, available for testing. Another drawback of this method is the fact that, especially with strips and grids, only the gyri are recorded and the activity in the depth of the sulci is not.

The main difference between cortical stimulation mapping (and the sodium amobarbital test) and the other mapping techniques is the fact that stimulation directly interferes with the language task under examination. Therefore, cortical stimulation identifies areas that are critical to language instead of highlighting active areas that are part of the language network for a given task but might not be essential (such as seen with nuclear medicine or fMRI).

Even though perioperative testing made stimulation more amenable to children and can now be performed by the bedside without restricting the child's movements significantly, it has again proved to be more difficult in children than in adults [54, 63-65]. Standard stimulation protocols used in adults had to be modified to obtain some response from the stimulation in children. Most studies use variations of the Jayakar et al. protocol [63] using rectangular biphasic pulses of current on two adjacent contacts, starting the stimulation at $1 \mathrm{~mA}$ with a $0.3 \mathrm{~ms}$ pulse of alternating polarity and a train duration of $3-5$ seconds. The stimulation intensity is then increased by steps of $1 \mathrm{~mA}$ and pulse duration by steps of $0.1-0.2 \mathrm{~ms}$ until after-discharges are seen, a seizure occurs, or there is a physiological response (speech arrest, motor, or sensory response) [63]. The various protocols use frequencies in the $20-50 \mathrm{~Hz}$ range, an intensity between 1 and $20 \mathrm{~mA}$, a pulse train 3-25 seconds in duration, and a pulse width between 0.14 and $0.2 \mathrm{~ms}$ [54, 66-68]. While intensities as low as $2-4 \mathrm{~mA}$ in adults generally evoke a response, in children, those intensities might have to be as high as $16-17 \mathrm{~mA}$ to be effective $[67,69-71]$.

The possible reasons for the difficulties encountered include incomplete myelination and the greater proportion of small fibers. The chronaxie, which is the pulse duration needed for stimulation to evoke a response, is directly affected by the myelin deposition, and increasing myelination leads to a decrease in time of chronaxie [63].

Schevon et al. found that 10.2 years was a cut-off age for successfully mapping cortex. He studied children with both the sodium amobarbital test and cortical stimulations and found that, before the age of $10,19 \%$ of children had positive cortical stimulation versus $87 \%$ for children older than 10
[54]. While a positive response is in general synonymous with critical language areas, having no response does not rule out critical language involvement in that area. The tasks administered during the stimulation are important, and, for example, expressive tasks show a better correlation with the sodium amobarbital test than receptive tasks [72]. However, within the expressive tasks, the generation of sentences might generate a larger perisylvian network of expressive and receptive language $[73,74]$ than a verb-generation task.

Various studies have used cortical stimulation to study intrahemispheric reorganization of language in epileptic patients. For example, Kadis et al. [75] demonstrated anterior reorganization of language in expressive language in the left frontal lobe.

4.5. $f M R I$. The basics of $\mathrm{fMRI}$ will not be discussed here because they are explained in another chapter of this issue (Wang et al.--Functional Magnetic Resonance Imaging for Language Mapping in TLE).

Once again, pediatric fMRI studies are more challenging than most adult fMRI studies. First, the cognitive level of the child is dependent on his age; therefore, the battery of tests should be age dependent. In addition, it is more difficult for a child than an adult to stay perfectly still in an MRI during acquisition of the task. Yuan showed a difference in age and gender in the motion of the head during acquisition; younger (5- to 9-year-old) male children moved the most. All groups moved less when engaged visually instead of just with an auditory stimuli (picture-word matching versus syntactic prosody, story processing, and verb generation) [16]. Another study showed a similar trend and demonstrated better results in children with normal developmental milestones as well as older children [76].

In addition, because children have smaller heads, the head coil should be adjusted for younger age groups. This modification is especially important for younger patients who, in addition to a small head have a shorter neck, would have their head in the lower quadrant of the coil if using an adult coil. In addition, the thickness of the skull changes with age, which influences the quality of the image. To adjust for all ages would require an institution to have different head size coils and to avoid surface coils that increase heterogeneity due to the thinner skull (signal is enhanced in thinner skulls of younger compared to older people) [77, 78]. Thinner skulls also produce increased physiological noise due to the increased heart and breathing frequency in children.

The entire MRI environment should be adapted to children. First, the addition of videos for viewing at the beginning of the acquisition, during the anatomical MRI as well as in between runs, would likely reduce motion artifact. Second, the buttons subjects push to answer questions should be adapted to smaller children's hands. Third, the child should be brought into the room and the magnet before the task without being rushed to acclimatize to the environment [77]; he/she should be fully prepared for the task and the environment before beginning the session [79].

Despite the above-mentioned problems, fMRI is starting to replace sodium amobarbital tests and cortical stimulation 
as a clinical tool for language mapping in some comprehensive epilepsy centres because it is less invasive, has a good spatial resolution $(1-3 \mathrm{~mm})$, and shows a good correlation with more invasive techniques [15, 72, 80-82].

fMRI can be used to investigate lateralization of language (laterality indexes are calculated based on the number of voxels activated on each side [82]) as well as to show a more precise localization of language areas. The areas with activated voxels are specific to the task being tested. As previously stated, there are various types of tasks that can be used, some active (verb generation, semantic decision, sentence completion, etc.) and some involving passive listening. The activations can be analyzed by contrasting any of these tasks with a resting state, during which the patient is instructed to do nothing, or between any of the conditions. The language map (pattern and lateralization) resulting from the analysis should differ depending on which tasks have been studied and contrasted [83].

Language protocols have to be developed especially for children and according to their age groups. In addition, delay in language development is common among epileptic children, which should be taken into account and tested before putting the child in the scanner.

fMRI shows the entire network of areas involved in the specific task (Figures 1 and 2). Because it is important to be as sensitive as possible to decrease the risk of postoperative deficit, paradigms capturing a wider network are usually preferred in pediatric populations (i.e., verb generation). While some studies have shown the possibility of performing 3-4 language tasks during image acquisition, so many tasks might be difficult in a clinical setting where the available time to train the child on the task and to remove him/her from the magnet in between tasks is less. However, one must be aware that only one language task might not be enough [84]. The authors illustrated with two case reports that hemispheric dissociation in language function is possible, which can only be detected when administering different types of language tasks (i.e., vowel identification tasks and "beep" story) [84].

The design of the study must be carefully developed to adequately capture the language network. Verb-generation tasks, in which the subject is asked to generate a maximum number of verbs related to a noun that is being presented (i.e., horse: jump, ride, etc.), seem to have a good reliability in determining hemispheric dominance $[41,82,85]$.

Story-processing tasks, in which the subject listens to a story with the instructions of listening carefully enough to be able to answer questions on each story after the MRI, seem to produce wider bilateral activation and show some asymmetries in pathological subjects $[86,87]$.

Another task commonly used in settings is a pictureword matching, in which the subject sees one or a couple of images and must decide whether it matches the name that is presented orally [42].

In younger children, a passive language (listening) task can also be used for patients between the ages of 2 and 4 years. However, it is difficult to control whether the subject is actually listening to the story as opposed to dreaming or sleeping [88].
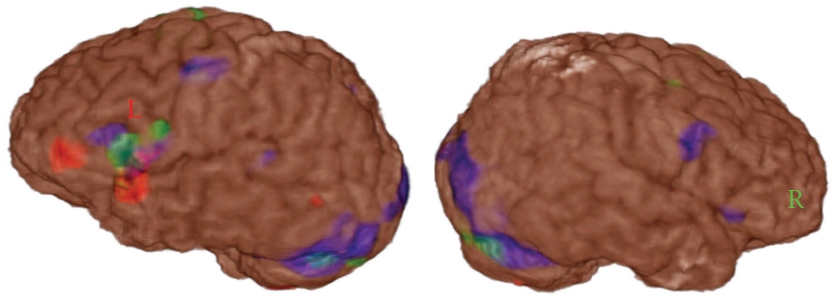

Figure 1: Example of fMRI with the superposition of different language tasks. While most of the tasks produce a left dominant hemispheric language activation, naming has a bilateral activation in the Broca area. Red: Verb generation, Green: sentence completion, Blue: naming.

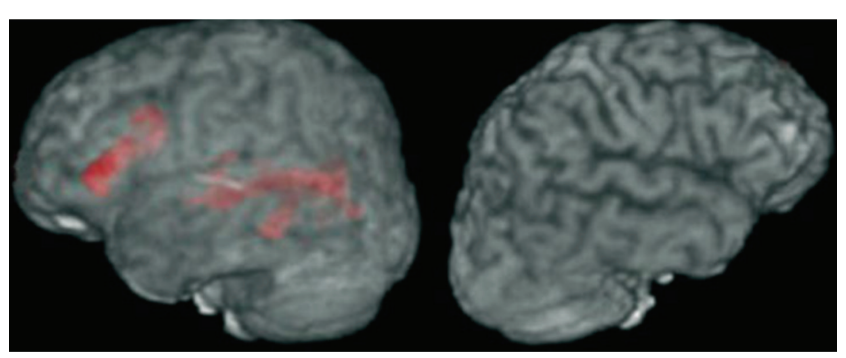

FIGURE 2: Another example of fMRI language activation map. This time the language map is solely left hemispheric in this sentence completion task.

In each task, different designs for each age group must be developed to provide an appropriate level of difficulty for the patient.

All tasks should include resting states or control tasks, such as button press, sensory test, or a finger-tapping task, to compare the task data to.

Additional unknowns remain when performing pediatric fMRI. The fMRI signal uses the hemodynamic response and the changes in oxyhemoglobin and deoxyhemoglobin to determine which areas of the brain are activated. Because cerebral blood flow varies across ages, should it be corrected for age when doing a group study? Does the immaturity of the brain affect the BOLD signal [88]? The grey/white matter ratio also varies greatly until the age of 7 years then continues to change over time [89]. The major fiber pathways are in place by the age of 3 , but the average density of neurons and synapses changes until the age of 16 , when it stabilizes until the late $60 \mathrm{~s}$. At the age of 7 , the average synaptic density of the frontal lobe is approximately 1.4-fold greater than that of an adult $[77,90]$. Do these developmental variations provide a stable enough environment to use the same methods of analysis when investigating a pediatric population? While most pediatric studies that have analyzed correlations with other modalities have found a good correlation, which indicates that fMRI seems to yield valid data even in children, those questions have to be considered.

The last caveat when using fMRI is analysis of data. The pediatric brain differs from an adult one, so group studies should not be normalized to an adult atlas, such as the Talairach atlas $[88,91,92]$. Recently, a pediatric atlas was 
developed and should be used instead [93]. This problem does not occur in a clinical setting in which the images of the patient are directly coregistered to his/her own anatomical MRI.

Careful analysis of the data is of paramount importance, keeping in mind that misused statistics can show anything. One should be sure of how the analysis has been performed and of the statistical value of the results before interpreting the data.

Similar to other functional imaging modalities, fMRI can be used to assess verbal and non-verbal memory. While in adults, memory mapping by fMRI is sometimes used as a clinical tool, it is usually part of a research protocol in the pediatric population. Because the hippocampus represents only a small area, the motion artifact and the difficulty of the tasks (without real-time feedback to know if the child is actually doing the task), it is difficult to have a valid study in a child.

While it is not as evident as using MEG, some studies have attempted to use fMRI to detect interictal spikes (spiketriggered fMRI) $[94,95]$.

\section{Emerging Techniques}

5.1. MEG. The creation of superconducting quantum interference devices (SQUIDs) in the late 1960s/early 1970s allowed a different method of recording of brain electrical activity [96]. MEG captures neuronal activity by recording the net current of the flow of ions, leading to an intracellular electrical current generating a magnetic flux. Repetitive events generate event-related potentials leading to evoked magnetic fields, which can then be recorded by the 248 channels positioned around the head. Because magnetic fields are less deformed than electrical fields on the scalp, the spatial resolution of the MEG $(2-4 \mathrm{~mm})$ is better than the EEG, and the images can be coregistered with an anatomical MRI to visualize them. In addition, because MEG measures neuronal activity, its temporal resolution is excellent $\left(10^{-3} \mathrm{sec}\right)$ [97].

In epileptic patients, MEG allows the recording of interictal spikes, sometimes seizures, and eloquent cortex.

Breier et al. demonstrated a good correlation between an MEG study and sodium amobarbital test in the pediatric population [98] such results have been reproduced in adults too [99]; Papanicolaou et al. [100] showed no differences in language pattern with age, but other studies on normal subjects comparing MEG to fMRI for receptive language mapping showed significant differences in language patterns between the two methods [101], and another study showed good concordance between MEG and fMRI lateralization in normal teenagers for picture verb-generation, but only $75 \%$ concordance for the word verb generation task, and even lower when looking at precise localization (voxel overlap $50 \%)$ [102]. In patients requiring surgery near languagerelated eloquent brain areas (mostly for tumors), a german group combined MEG and fMRI and showed a good congruence between the two modalities (96\%); however for some patient language activation was only seen in one of the modality [103]. MEG is still in its infancy when considering language mapping in epileptic patients, and more studies are required to see how it can be used in presurgical planning. The National Institute of Neurological Disorders and Stroke is currently recruiting patients for a large trial (NCT00706160).

5.2. fNIRS. Near-infrared spectroscopy is a noninvasive technique used to measure hemodynamic changes using the different light absorption spectra of oxyhemoglobin ( $\mathrm{HbO}$ ) and deoxyhemoglobin (HbR). It necessitates a light-emitting source (at two different wavelengths, usually between 680 and $1000 \mathrm{~nm}$ ), a detector, and a dispersive element. This method is also called optical tomography because it uses an exogenous optical tracer to extrapolate the blood flow, blood volume, or oxygenation of a specific region of the brain. For example, it can be used in conjunction with a bolus injection of indocyanine green to measure cerebral blood flow. It is also used to detect changes produced by neuronal activity (similarly to fMRI). When there is regional activation during a task, there is an "initial dip" with a reduction of $\mathrm{HbO}$ and an increase of $\mathrm{HbR}$, then a large increase of $\mathrm{HbO}$ and a decrease of HbR (focal arterial blood flow). Because of the shallow penetration of photons ( $3-5 \mathrm{~cm}$ below the scalp), the activity of the cortex can be monitored but not activity of deeper structures [104].

The advantage of fNIRS is that it does not restrict the child to a small space such as an MRI scanner does, and because the device is directly on the head of the child, motion artifacts are not a problem. The other advantage of fNIRS is that the child can actually speak during the task; therefore, his understanding and involvement in the task are actively monitored.

fNIRS seems to be more sensitive to bilateral speech pattern, which is sometimes more difficult to analyze with fMRI, as shown by Benke et al. [105], when there is a dissociation between frontal and temporal activations [104, $106,107]$. However, fNIRS is currently only used in research and in some specific centers.

5.3. DTI. Diffusion tensor imaging is a technique that enables tracing of neuronal tracts in the brain. Because of the tubular nature of neurons, water can move freely in the direction of the axis but is restricted transversally by the membrane. When applying various field gradients to the brain in the MR scanner, the difference between the diffusivity in the two axes can be represented by a tensor and subsequently mapped to the brain by coregistering it with an anatomical image to obtain mean diffusivity and fractional anisotropy maps. These maps can aid in the understanding of functional connectivity of the brain by displaying fibers connecting two regions $[108,109]$.

There are currently no pediatric studies investigating the use of DTI and fMRI together for language mapping. However, in adults, a few groups have tried to predict language lateralization by studying the arcuate fasciculus, inferior longitudinal fasciculus, or uncinate fasciculus and their asymmetry reflected by the anisotropy value [110], the 
association between anisotropic or mean diffusivity values and language deficits in patients with TLE [111], or finally with DTI and cortical mapping to assess colocalization of language areas in the anterior and posterior part of the arcuate fasciculus. A number of studies have shown that increased mean diffusivity and decreased fractional anisotropy, interpreted as structural compromise of the white matter tracts, are associated with language deficits in patients with epilepsy [111-114].

5.4. TMS. Transcranial magnetic stimulation (TMS) is a noninvasive technique, which uses focal magnetic field generated by a rapidly changing current within a conducting coil. The coil can be applied to the scalp, so the magnetic field has a direct effect on the brain by depolarizing or hyperpolarizing neurons. Experiments have shown that TMS can induce a transient change in behavior by interfering in a manner similar to cortical stimulation. There are several methods for delivering the magnetic field, including singlepulse, paired pulse, and repetitive pulses.

A first study on language and TMS was performed in adults by Pascual-Leone et al. [115], who was able to induce speech arrest when stimulating the perisylvian cortex with 10-s trains of repetitive TMS (rTMS) applied at rates of 8$25 \mathrm{~Hz}$. Variations in the frequency of the stimulation were tried, and speech was also disrupted at $4 \mathrm{~Hz}$ in another study [116]. The same group showed a good correlation between sodium amobarbital test and TMS in 12/16 epilepsy patients [117], while other studies showed better even better correlations $[115,118]$.

While some studies have been performed in children and one review article described the safety of TMS in children (including a potential for increased risk of seizures in children younger than 5 years [119]), there are no language studies in this group of patients. Some motor studies have shown that it is feasible [120]; however, recommendations state that TMS should be avoided in young children [119].

\section{Conclusion}

Language mapping in children requires a specialized multidisciplinary team with specific protocols designed from a developmental perspective. However, an increasing number of noninvasive techniques have been shown to be reliable and are being implemented clinically in preoperative investigation for epileptic children.

\section{References}

[1] B. F. Bourgeois, "Temporal lobe epilepsy in infants and children," Brain and Development, vol. 20, no. 3, pp. 135-141, 1998.

[2] A. Ray and P. Kotagal, "Temporal lobe epilepsy in children: overview of clinical semiology," Epileptic Disorders, vol. 7, no. 4, pp. 299-307, 2005.

[3] C. Bocti, Y. Robitaille, P. Diadori et al., "The pathological basis of temporal lobe epilepsy in childhood," Neurology, vol. 60, no. 2, pp. 191-195, 2003.
[4] Y. J. Lee, H. C. Kang, S. J. Bae et al., "Comparison of temporal lobectomies of children and adults with intractable temporal lobe epilepsy," Child's Nervous System, vol. 26, no. 2, pp. 177183, 2010.

[5] D. B. Sinclair, M. Wheatley, K. Aronyk et al., "Pathology and neuroimaging in pediatric temporal lobectomy for intractable epilepsy," Pediatric Neurosurgery, vol. 35, no. 5, pp. 239-246, 2001.

[6] O. Vernet, J. P. Farmer, J. L. Montes, J. G. Villemure, and K. Meagher-Villemure, "Dysgenetic mesial temporal sclerosis: an unrecognized entity," Child's Nervous System, vol. 16, no. 10-11, pp. 719-723, 2000.

[7] H. Clusmann, T. Kral, E. Fackeldey et al., "Lesional mesial temporal lobe epilepsy and limited resections: prognostic factors and outcome," Journal of Neurology, Neurosurgery and Psychiatry, vol. 75, no. 11, pp. 1589-1596, 2004.

[8] M. Lendt, U. Gleissner, C. Helmstaedter, R. Sassen, H. Clusmann, and C. E. Elger, "Neuropsychological outcome in children after frontal lobe epilepsy surgery," Epilepsy and Behavior, vol. 3, no. 1, pp. 51-59, 2002.

[9] D. B. Sinclair, K. Aronyk, T. Snyder et al., "Pediatric temporal lobectomy for epilepsy," Pediatric Neurosurgery, vol. 38, no. 4, pp. 195-205, 2003.

[10] M. Lassonde, H. C. Sauerwein, I. Jambaqué, M. L. Smith, and C. Helmstaedter, "Neuropsychology of childhood epilepsy: pre- and postsurgical assessment," Epileptic Disorders, vol. 2, no. 1, pp. 3-13, 2000.

[11] B. Abou-Khalil, "Methods for determination of language dominance: the wada test and proposed noninvasive alternatives," Current Neurology and Neuroscience Reports, vol. 7, no. 6, pp. 483-490, 2007.

[12] J. Pujol, J. Deus, J. M. Losilla, and A. Capdevila, "Cerebral lateralization of language in normal left-handed people studied by functional MRI," Neurology, vol. 52, no. 5, pp. 1038-1043, 1999.

[13] J. P. Szaflarski, J. R. Binder, E. T. Possing, K. A. McKiernan, B. D. Ward, and T. A. Hammeke, "Language lateralization in left-handed and ambidextrous people: fMRI data," Neurology, vol. 59, no. 2, pp. 238-244, 2002.

[14] X. You, M. Adjouadi, M. R. Guillen et al., "Sub-patterns of language network reorganization in pediatric localization related epilepsy: a multisite study," Human Brain Mapping, vol. 32, no. 5, pp. 784-799, 2011.

[15] F. G. Woermann, H. Jokeit, R. Luerding et al., "Language lateralization by Wada test and fMRI in 100 patients with epilepsy," Neurology, vol. 61, no. 5, pp. 699-701, 2003.

[16] W. Yuan, J. P. Szaflarski, V. J. Schmithorst et al., "fMRI shows atypical language lateralization in pediatric epilepsy patients," Epilepsia, vol. 47, no. 3, pp. 593-600, 2006.

[17] J. A. Springer, J. R. Binder, T. A. Hammeke et al., "Language dominance in neurologically normal and epilepsy subjects: a functional MRI study," Brain, vol. 122, no. 11, pp. 2033-2045, 1999.

[18] D. K. Binder and J. Schramm, "Transsylvian functional hemispherectomy," Child's Nervous System, vol. 22, no. 8, pp. 960966, 2006.

[19] W. D. Gaillard, L. Balsamo, B. Xu et al., "Language dominance in partial epilepsy patients identified with an fMRI reading task," Neurology, vol. 59, no. 2, pp. 256-265, 2002.

[20] W. D. Gaillard, L. Balsamo, B. Xu et al., "fMRI language task panel improves determination of language dominance," Neurology, vol. 63, no. 8, pp. 1403-1408, 2004. 
[21] W. D. Gaillard, M. M. Berl, E. N. Moore et al., "Atypical language in lesional and nonlesional complex partial epilepsy," Neurology, vol. 69, no. 18, pp. 1761-1771, 2007.

[22] N. F. Ramsey, I. E. C. Sommer, G. J. Rutten, and R. S. Kahn, "Combined analysis of language tasks in fMRI improves assessment of hemispheric dominance for language functions in individual subjects," NeuroImage, vol. 13, no. 4, pp. 719733, 2001.

[23] L. R. Rosenberger, J. Zeck, M. M. Berl et al., "Interhemispheric and intrahemispheric language reorganization in complex partial epilepsy," Neurology, vol. 72, no. 21, pp. 1830-1836, 2009.

[24] M. Staudt, W. Grodd, G. Niemann, D. Wildgruber, M. Erb, and I. Krägeloh-Mann, "Early left periventricular brain lesions induce right hemispheric organization of speech," Neurology, vol. 57, no. 1, pp. 122-125, 2001.

[25] M. Staudt, K. Lidzba, W. Grodd, D. Wildgruber, M. Erb, and I. Krägeloh-Mann, "Right-hemispheric organization of language following early left-sided brain lesions: functional MRI topography," NeuroImage, vol. 16, no. 4, pp. 954-967, 2002.

[26] J. Saltzman, M. L. Smith, and K. Scott, "The impact of age at seizure onset on the likelihood of atypical language representation in children with intractable epilepsy," Brain and Cognition, vol. 48, no. 2-3, pp. 517-520, 2002.

[27] M. Brázdil, P. Chlebus, M. Mikl, M. Pažourková, P. Krupa, and I. Rektor, "Reorganization of language-related neuronal networks in patients with left temporal lobe epilepsy-an fMRI study," European Journal of Neurology, vol. 12, no. 4, pp. 268-275, 2005.

[28] J. Janszky, A. Ebner, B. Kruse et al., "Functional organization of the brain with malformations of cortical development," Annals of Neurology, vol. 53, no. 6, pp. 759-767, 2003.

[29] J. Janszky, H. Jokeit, D. Heinemann, R. Schulz, F. G. Woermann, and A. Ebner, "Epileptic activity influences the speech organization in medial temporal lobe epilepsy," Brain, vol. 126, no. 9, pp. 2043-2051, 2003.

[30] L. Thivard, J. Hombrouck, S. Tézenas Du Montcel et al., "Productive and perceptive language reorganization in temporal lobe epilepsy," NeuroImage, vol. 24, no. 3, pp. 841851, 2005.

[31] D. P. Anderson, A. S. Harvey, M. M. Saling et al., "fMRI lateralization of expressive language in children with cerebral lesions," Epilepsia, vol. 47, no. 6, pp. 998-1008, 2006.

[32] G. Dehaene-Lambertz, S. Dehaene, and L. Hertz-Pannier, "Functional neuroimaging of speech perception in infants," Science, vol. 298, no. 5600, pp. 2013-2015, 2002.

[33] F. Homae, H. Watanabe, T. Nakano, K. Asakawa, and G. Taga, "The right hemisphere of sleeping infant perceives sentential prosody," Neuroscience Research, vol. 54, no. 4, pp. 276-280, 2006.

[34] T. Imada, Y. Zhang, M. Cheour, S. Taulu, A. Ahonen, and P. K. Kuhl, "Infant speech perception activates Broca's area: a developmental magnetoencephalography study," NeuroReport, vol. 17, no. 10, pp. 957-962, 2006.

[35] I. Wartenburger, J. Steinbrink, S. Telkemeyer, M. Friedrich, A. D. Friederici, and H. Obrig, "The processing of prosody: evidence of interhemispheric specialization at the age of four," NeuroImage, vol. 34, no. 1, pp. 416-425, 2007.

[36] S. Holland, J. Vannest, M. Mecoli et al., "Functional MRI of language lateralization during development in children," International Journal of Audiology, vol. 46, no. 9, pp. 533-551, 2007.
[37] V. Ressel, M. Wilke, K. Lidzba, W. Lutzenberger, and I. Krägeloh-Mann, "Increases in language lateralization in normal children as observed using magnetoencephalography," Brain and Language, vol. 106, no. 3, pp. 167-176, 2008.

[38] F. Vargha-Khadem, A. M. O'Gorman, and G. V. Watters, "Aphasia and handedness in relation to hemispheric side, age at injury and severity of cerebral lesion during childhood," Brain, vol. 108, no. 3, pp. 677-696, 1985.

[39] R. A. Müller, H. T. Chugani, O. Muzik, and T. J. Mangner, "Brain organization of motor and language functions following hemispherectomy: a $\left[{ }^{15} \mathrm{O}\right]$-water positron emission tomography study," Journal of Child Neurology, vol. 13, no. 1, pp. $16-22,1998$.

[40] R. A. Müller, M. E. Behen, R. D. Rothermel, O. Muzik, P. K. Chakraborty, and H. T. Chugani, "Brain organization for language in children, adolescents, and adults with left hemisphere lesion: a PET study," Progress in NeuroPsychopharmacology and Biological Psychiatry, vol. 23, no. 4, pp. 657-668, 1999.

[41] S. K. Holland, E. Plante, A. Weber Byars, R. H. Strawsburg, V. J. Schmithorst, and W. S. Ball, "Normal fMRI brain activation patterns in children performing a verb generation task," NeuroImage, vol. 14, no. 4, pp. 837-843, 2001.

[42] V. J. Schmithorst, S. K. Holland, and E. Plante, "Object identification and lexical/semantic access in children: a functional magnetic resonance imaging study of word-picture matching," Human Brain Mapping, vol. 28, no. 10, pp. 10601074, 2007.

[43] J. Vannest, P. R. Karunanayaka, M. Altaye et al., "Comparison of fMRI data from passive listening and active-response story processing tasks in children," Journal of Magnetic Resonance Imaging, vol. 29, no. 4, pp. 971-976, 2009.

[44] I. Baron, "Maxims and a model for the practice of pediatric neuropsychology," in Pediatric Neuropsychology. Research Theory and Practice, K. O. Yeates et al., Ed., pp. 472-498, Guilford Press, New York, NY, USA, 2010.

[45] M. A. Fernandes and M. L. Smith, "Comparing the Fused Dichotic Words Test and the Intracarotid Amobarbital Procedure in children with epilepsy," Neuropsychologia, vol. 38, no. 9, pp. 1216-1228, 2000.

[46] K. Hugdahl, G. Carlsson, P. Uvebrant, and A. J. Lundervold, "Dichotic-listening performance and intracarotid injections of amobarbital in children and adolescents: preoperative and postoperative comparisons," Archives of Neurology, vol. 54, no. 12, pp. 1494-1500, 1997.

[47] R. J. Zatorre, "Perceptual asymmetry on the dichotic fused words test and cerebral speech lateralization determined by the carotid sodium Amytal test," Neuropsychologia, vol. 27, no. 10, pp. 1207-1219, 1989.

[48] M. R. Rosenweig, "Representations of the two ears at the auditory cortex," The American journal of physiology, vol. 167, no. 1, pp. 147-158, 1951.

[49] T. de Koning, H. Versnel, A. Jennekens-Schinkel et al., "Language development before and after temporal surgery in children with intractable epilepsy," Epilepsia, vol. 50, no. 11, pp. 2408-2419, 2009.

[50] P. Rankin and F. Vargha-Khadem, "Neuropsychological evaluation-children," in Epilepsy: A Comprehensive Textbook, J. Engel and T. A. Pedley, Eds., Lippincott Williams \& Wilkins, Philadelphia, Pa, USA, 2007.

[51] M. Catani, D. K. Jones, and D. H. Ffytche, "Perisylvian language networks of the human brain," Annals of Neurology, vol. 57, no. 1, pp. 8-16, 2007. 
[52] G. P. Lee, M. Westerveld, L. B. Blackburn, Y. D. Park, and D. W. Loring, "Prediction of verbal memory decline after epilepsy surgery in children: effectiveness of Wada memory asymmetries," Epilepsia, vol. 46, no. 1, pp. 97-103, 2005.

[53] C. A. Szabo and E. Wyllie, "Intracarotid amobarbital testing for language and memory dominance in children," Epilepsy Research, vol. 15, no. 3, pp. 239-246, 1993.

[54] C. Schevon, C. Carlson, C. M. Zaroff et al., "Pediatric language mapping: sensitivity of neurostimulation and Wada testing in epilepsy surgery," Epilepsia, vol. 48, no. 3, pp. 539$545,2007$.

[55] H. M. Hamer, E. Wyllie, L. Stanford, E. Mascha, P. Kotagal, and B. Wolgamuth, "Risk factors for unsuccessful testing during the intracarotid amobarbital procedure in preadolescent children," Epilepsia, vol. 41, no. 5, pp. 554-563, 2000.

[56] F. C. Vinas, L. Zamorano, R. A. Mueller et al., "[15O]-water PET and intraoperative brain mapping: a comparison in the localization of eloquent cortex," Neurological Research, vol. 19, no. 6, pp. 601-608, 1997.

[57] R. Tatlidil, J. Xiong, and S. Luther, "Presurgical lateralization of seizure focus and language dominant hemisphere with $\mathrm{O}$ 15 water PET imaging," Acta Neurologica Scandinavica, vol. 102, no. 2, pp. 73-80, 2000.

[58] K. Borbély, A. Gjedde, I. Nyáry, S. Czirják, N. Donauer, and A. Buck, "Speech activation of language dominant hemisphere: a single-photon emission computed tomography study," NeuroImage, vol. 20, no. 2, pp. 987-994, 2003.

[59] J. D. Duncan, S. David Moss, D. J. Bandy et al., "Use of positron emission tomography for presurgical localization of eloquent brain areas in children with seizures," Pediatric Neurosurgery, vol. 26, no. 3, pp. 144-156, 1997.

[60] E. L. So, "Integration of EEG, MRI, and SPECT in localizing the seizure focus for epilepsy surgery," Epilepsia, vol. 41, supplement 3, pp. S48-S54, 2000.

[61] O. Willmann, R. Wennberg, T. May, F. G. Woermann, and B. Pohlmann-Eden, "The contribution of 18F-FDG PET in preoperative epilepsy surgery evaluation for patients with temporal lobe epilepsy. A meta-analysis," Seizure, vol. 16, no. 6, pp. 509-520, 2007.

[62] W. Penfield, "Combined regional and general anesthesia for craniotomy and cortical exploration. I. Neurosurgical considerations," Current Researches in Anesthesia and Analgesia, vol. 33, no. 3, pp. 145-155, 1954.

[63] P. Jayakar, L. A. Alvarez, M. S. Duchowny, and T. J. Resnick, "A safe and effective paradigm to functionally map the cortex in childhood," Journal of Clinical Neurophysiology, vol. 9, no. 2, pp. 288-293, 1992.

[64] P. Jayakar, M. Duchowny, and T. Resnick, "Subdural monitoring in the evaluation of children for epilepsy surgery," Journal of Child Neurology, vol. 9, no. 2, pp. 61-66, 1994.

[65] S. G. Ojemann, M. S. Berger, E. Lettich, and G. A. Ojemann, "Localization of language function in children: results of electrical stimulation mapping," Journal of Neurosurgery, vol. 98, no. 3, pp. 465-470, 2003.

[66] W. B. Gallentine and M. A. Mikati, "Intraoperative electrocorticography and cortical stimulation in children," Journal of Clinical Neurophysiology, vol. 26, no. 2, pp. 95-108, 2009.

[67] G. J. Rutten, P. C. van Rijen, C. W. M. van Veelen, and N. F. Ramsey, "Language area localization with three-dimensional functional magnetic resonance imaging matches intrasulcal electrostimulation in Broca's area," Annals of Neurology, vol. 46, no. 3, pp. 405-408, 1999.
[68] F. Signorelli, J. Guyotat, C. Mottolese, F. Schneider, G. D'Acunzi, and J. Isnard, "Intraoperative electrical stimulation mapping as an aid for surgery of intracranial lesions involving motor areas in children," Child's Nervous System, vol. 20, no. 6, pp. 420-426, 2004.

[69] D. B. FitzGerald, G. R. Cosgrove, S. Ronner et al., "Location of language in the cortex: a comparison between functional MR imaging and electrocortical stimulation," American Journal of Neuroradiology, vol. 18, no. 8, pp. 1529-1539, 1997.

[70] P. Marusic, I. M. Najm, Z. Ying et al., "Focal cortical dysplasias in eloquent cortex: functional characteristics and correlation with MRI and histopathologic changes," Epilepsia, vol. 43, no. 1, pp. 27-32, 2002.

[71] F. Z. Yetkin, W. M. Mueller, G. L. Morris et al., "Functional MR activation correlated with intraoperative cortical mapping," American Journal of Neuroradiology, vol. 18, no. 7, pp. 1311-1315, 1997.

[72] S. Lehéricy, L. Cohen, B. Bazin et al., "Functional MR evaluation of temporal and frontal language dominance compared with the Wada test," Neurology, vol. 54, no. 8, pp. 1625-1633, 2000.

[73] R. A. Müller, R. D. Rothermel, M. E. Behen, O. Muzik, T. J. Mangner, and H. T. Chugani, "Receptive and expressive language activations for sentences: a PET study," NeuroReport, vol. 8, no. 17, pp. 3767-3770, 1997.

[74] M. Vigneau, V. Beaucousin, P. Y. Hervé et al., "Metaanalyzing left hemisphere language areas: phonology, semantics, and sentence processing," NeuroImage, vol. 30, no. 4, pp. 1414-1432, 2006.

[75] D. S. Kadis, K. Iida, E. N. Kerr et al., "Intrahemispheric reorganization of language in children with medically intractable epilepsy of the left hemisphere," Journal of the International Neuropsychological Society, vol. 13, no. 3, pp. 505-516, 2007.

[76] B. E. Yerys, K. F. Jankowski, D. Shook et al., "The fMRI success rate of children and adolescents: typical development, epilepsy, attention deficit/hyperactivity disorder, and autism spectrum disorders," Human Brain Mapping, vol. 30, no. 10, pp. 3426-3435, 2009.

[77] E. Kotsoni, D. Byrd, and B. Casey, "Special considerations for functional magnetic resonance imaging of pediatric populations," Journal of Magnetic Resonance Imaging, vol. 23, no. 6, pp. 877-886, 2006.

[78] K. M. Thomas, S. W. King, P. L. Franzen et al., "A developmental functional MRI study of spatial working memory," NeuroImage, vol. 10, no. 3, pp. 327-338, 1999.

[79] H. Shurtleff, M. Warner, A. Poliakov et al., "Functional magnetic resonance imaging for presurgical evaluation of very young pediatric patients with epilepsy," Journal of Neurosurgery: Pediatrics, vol. 5, no. 5, pp. 500-506, 2010.

[80] L. M. Balsamo and W. D. Gaillard, "The utility of functional magnetic resonance imaging in epilepsy and language," Current Neurology and Neuroscience Reports, vol. 2, no. 2, pp. 142-149, 2002.

[81] J. R. Binder, S. J. Swanson, T. A. Hammeke et al., "Determination of language dominance using functional MRI: a comparison with the Wada test," Neurology, vol. 46, no. 4, pp. 978-984, 1996.

[82] L. Hertz-Pannier, W. D. Gaillard, S. H. Mott et al., "Noninvasive assessment of language dominance in children and adolescents with functional MRI: a preliminary study," Neurology, vol. 48, no. 4, pp. 1003-1012, 1997.

[83] J. R. Binder, S. J. Swanson, T. A. Hammeke, and D. S. Sabsevitz, "A comparison of five fMRI protocols for mapping 
speech comprehension systems," Epilepsia, vol. 49, no. 12, pp. 1980-1997, 2008.

[84] M. Wilke, T. Pieper, K. Lindner, T. Dushe, H. Holthausen, and I. Krägeloh-Mann, "Why one task is not enough: functional MRI for atypical language organization in two children," European Journal of Paediatric Neurology, vol. 14, no. 6, pp. 474-478, 2010.

[85] F. Liégeois, J. H. Cross, D. G. Gadian, and A. Connelly, "Role of fMRI in decision-making process: epilepsy surgery for children," Journal of Magnetic Resonance Imaging, vol. 23, no. 6, pp. 933-940, 2006.

[86] P. R. Karunanayaka, S. K. Holland, V. J. Schmithorst et al., "Age-related connectivity changes in fMRI data from children listening to stories," NeuroImage, vol. 34, no. 1, pp. 349-360, 2007.

[87] M. Wilke, S. K. Holland, and W. S. Ball Jr., "Language processing during natural sleep in a 6-year-old boy, as assessed with functional MR imaging," American Journal of Neuroradiology, vol. 24, no. 1, pp. 42-44, 2003.

[88] E. Freilich and W. Gaillard, "Utility of functional MRI in pediatric neurology," Current Neurology and Neuroscience Reports, vol. 10, no. 1, pp. 40-46, 2010.

[89] E. Courchesne, H. J. Chisum, J. Townsend et al., "Normal brain development and aging: quantitative analysis at in vivo MR imaging in healthy volunteers," Radiology, vol. 216, no. 3, pp. 672-682, 2000.

[90] J. Matsuzawa, M. Matsui, T. Konishi et al., "Age-related volumetric changes of brain gray and white matter in healthy infants and children," Cerebral Cortex, vol. 11, no. 4, pp. 335$342,2001$.

[91] O. Muzik, D. C. Chugani, C. Juhăsz, C. Shen, and H. T. Chugani, "Statistical parametric mapping: assessment of application in children," NeuroImage, vol. 12, no. 5, pp. 538$549,2000$.

[92] M. Wilke, V. J. Schmithorst, and S. K. Holland, "Assessment of spatial normalization of whole-brain magnetic resonance images in children," Human Brain Mapping, vol. 17, no. 1, pp. 48-60, 2002.

[93] M. Wilke, S. K. Holland, M. Altaye, and C. Gaser, "TemplateO-Matic: a toolbox for creating customized pediatric templates," NeuroImage, vol. 41, no. 3, pp. 903-913, 2008.

[94] A. Al-Asmi, C. G. Bénar, D. W. Gross et al., "fMRI activation in continuous and spike-triggered EEG-fMRI studies of epileptic spikes," Epilepsia, vol. 44, no. 10, pp. 1328-1339, 2003.

[95] J. S. Archer, R. S. Briellman, D. F. Abbott, A. Syngeniotis, R. M. Wellard, and G. D. Jackson, "Benign epilepsy with centrotemporal spikes: spike triggered fMRI shows somato-sensory cortex activity," Epilepsia, vol. 44, no. 2, pp. 200-204, 2003.

[96] D. Cohen, "Magnetoencephalography: detection of the brain's electrical activity with a superconducting magnetometer," Science, vol. 175, no. 4022, pp. 664-666, 1972.

[97] R. Grondin, S. Chuang, H. Otsubo et al., "The role of magnetoencephalography in pediatric epilepsy surgery," Child's Nervous System, vol. 22, no. 8, pp. 779-785, 2006.

[98] J. I. Breier, R. Billingsley-Marshall, E. Pataraia, E. M. Castillo, and A. C. Papanicolaou, "Magnetoencephalographic studies of language reorganization after cerebral insult," Archives of Physical Medicine and Rehabilitation, vol. 87, supplement 2, no. 12, pp. S77-S83, 2006.

[99] M. Pirmoradi, R. Béland, D. K. Nguyen, B. A. Bacon, and M. Lassonde, "Language tasks used for the presurgical assessment of epileptic patients with MEG," Epileptic Disorders, vol. 12, no. 2, pp. 97-108, 2010.
[100] A. C. Papanicolaou, P. Pazo-Alvarez, E. M. Castillo et al., "Functional neuroimaging with MEG: normative language profiles," NeuroImage, vol. 33, no. 1, pp. 326-342, 2006.

[101] R. L. Billingsley-Marshall, T. Clear, W. E. Mencl et al., "A comparison of functional MRI and magnetoencephalography for receptive language mapping," Journal of Neuroscience Methods, vol. 161, no. 2, pp. 306-313, 2007.

[102] E. W. Pang, F. Wang, M. Malone, D. S. Kadis, and E. J. Donner, "Localization of Broca's area using verb generation tasks in the MEG: validation against fMRI," Neuroscience Letters, vol. 490, no. 3, pp. 215-219, 2011.

[103] P. Grummich, C. Nimsky, E. Pauli, M. Buchfelder, and O. Ganslandt, "Combining fMRI and MEG increases the reliability of presurgical language localization: a clinical study on the difference between and congruence of both modalities," NeuroImage, vol. 32, no. 4, pp. 1793-1803, 2006.

[104] V. Quaresima, S. Bisconti, and M. Ferrari, "A brief review on the use of functional near-infrared spectroscopy (fNIRS) for language imaging studies in human newborns and adults," Brain and Language. In press.

[105] T. Benke, B. Köylü, P. Visani et al., "Language lateralization in temporal lobe epilepsy: a comparison between fMRI and the Wada Test," Epilepsia, vol. 47, no. 8, pp. 1308-1319, 2006.

[106] A. Gallagher, R. Beland, and M. Lassonde, "The contribution of functional near-infrared spectroscopy (fNIRS) to the presurgical assessment of language function in children," Brain and Language. In press.

[107] I. Kovelman, M. H. Shalinsky, K. S. White et al., "Dual language use in sign-speech bimodal bilinguals: fNIRS brainimaging evidence," Brain and Language, vol. 109, no. 2-3, pp. 112-123, 2009.

[108] P. J. Basser and D. K. Jones, "Diffusion-tensor MRI: theory, experimental design and data analysis-a technical review," NMR in Biomedicine, vol. 15, no. 7-8, pp. 456-467, 2002.

[109] P. Sundgren, Q. Dong, D. Gómez-Hassan, S. K. Mukherji, P. Maly, and R. Welsh, "Diffusion tensor imaging of the brain: review of clinical applications," Neuroradiology, vol. 46, no. 5, pp. 339-350, 2004.

[110] T. M. Ellmore, M. S. Beauchamp, J. I. Breier et al., “Temporal lobe white matter asymmetry and language laterality in epilepsy patients," NeuroImage, vol. 49, no. 3, pp. 2033-2044, 2010.

[111] C. R. McDonald, M. E. Ahmadi, D. J. Hagler et al., "Diffusion tensor imaging correlates of memory and language impairments in temporal lobe epilepsy," Neurology, vol. 71, no. 23, pp. 1869-1876, 2008.

[112] B. Diehl, R. M. Busch, J. S. Duncan, Z. Piao, J. Tkach, and H. O. Lüders, "Abnormalities in diffusion tensor imaging of the uncinate fasciculus relate to reduced memory in temporal lobe epilepsy," Epilepsia, vol. 49, no. 8, pp. 1409-1418, 2008.

[113] H. W. Powell, G. J. M. Parker, D. C. Alexander et al., "Imaging language pathways predicts postoperative naming deficits," Journal of Neurology, Neurosurgery and Psychiatry, vol. 79, no. 3, pp. 327-330, 2008.

[114] H. W. Powell, G. J. M. Parker, D. C. Alexander et al., "Abnormalities of language networks in temporal lobe epilepsy," NeuroImage, vol. 36, no. 1, pp. 209-221, 2007.

[115] A. Pascual-Leone, J. R. Gates, and A. Dhuna, "Induction of speech arrest and counting errors with rapid-rate transcranial magnetic stimulation," Neurology, vol. 41, no. 5, pp. 697702, 1991.

[116] C. M. Epstein, K. J. Meador, D. W. Loring et al., "Localization and characterization of speech arrest during transcranial 
magnetic stimulation," Clinical Neurophysiology, vol. 110, no. 6, pp. 1073-1079, 1999.

[117] C. M. Epstein, J. L. Woodard, A. Y. Stringer et al., "Repetitive transcranial magnetic stimulation does not replicate the Wada test," Neurology, vol. 55, no. 7, pp. 1025-1027, 2000.

[118] P. Jennum, L. Friberg, A. Fuglsang-Frederiksen, and M. Dam, "Speech localization using repetitive transcranial magnetic stimulation," Neurology, vol. 44, no. 2, pp. 269-273, 1994.

[119] E. M. Wassermann, "Risk and safety of repetitive transcranial magnetic stimulation: report and suggested guidelines from the International Workshop on the Safety of Repetitive Transcranial Magnetic Stimulation, June 5-7, 1996," Electroencephalography and Clinical Neurophysiology, vol. 108, no. 1, pp. 1-16, 1998.

[120] A. Nezu, S. Kimura, S. Uehara, T. Kobayashi, M. Tanaka, and K. Saito, "Magnetic stimulation of motor cortex in children: maturity of corticospinal pathway and problem of clinical application," Brain and Development, vol. 19, no. 3, pp. 176180, 1997. 


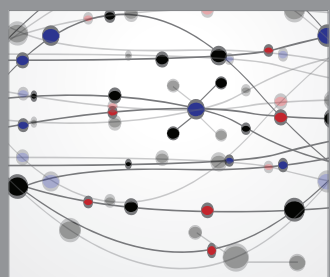

The Scientific World Journal
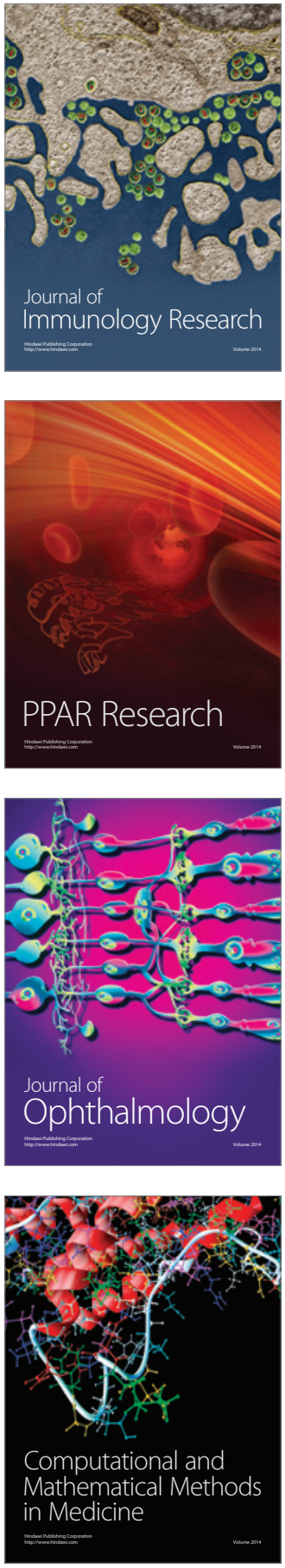

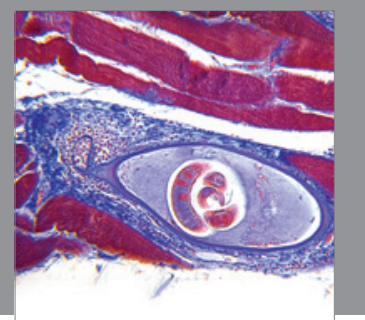

Gastroenterology

Research and Practice
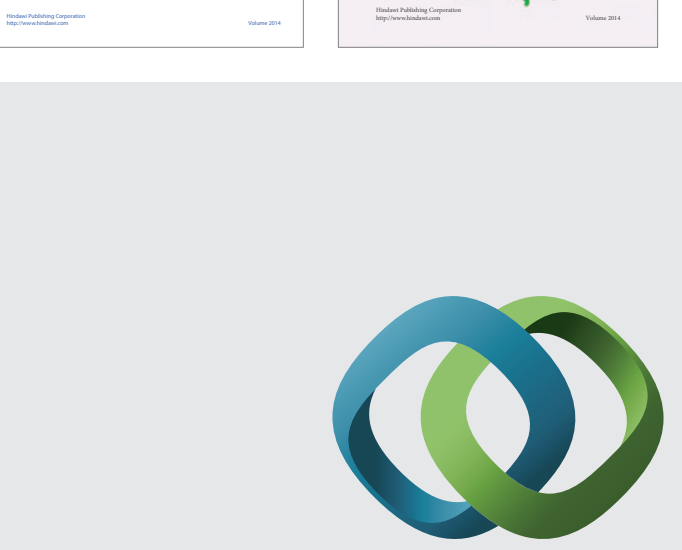

\section{Hindawi}

Submit your manuscripts at

http://www.hindawi.com
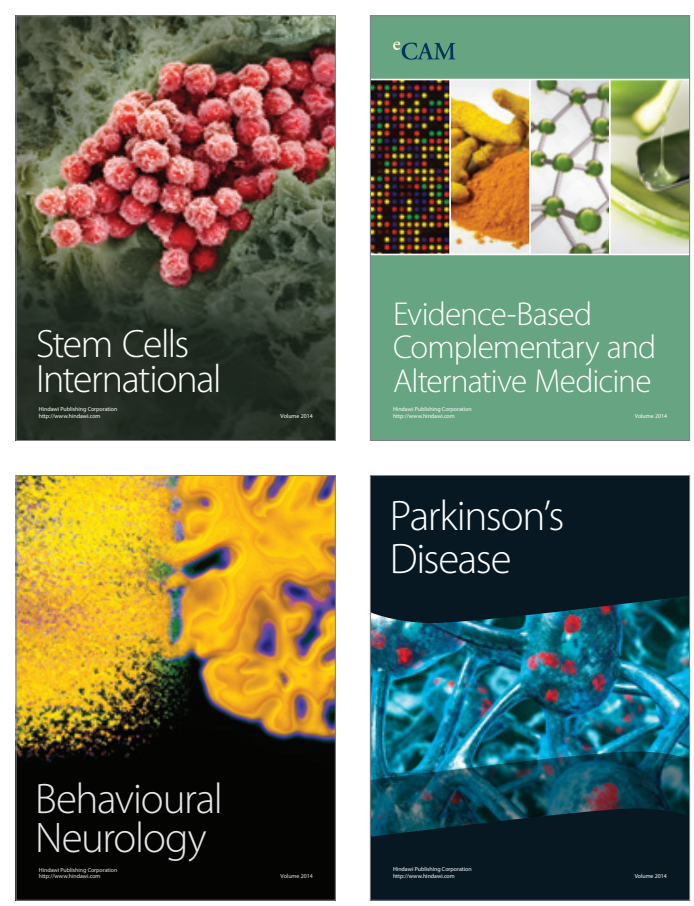

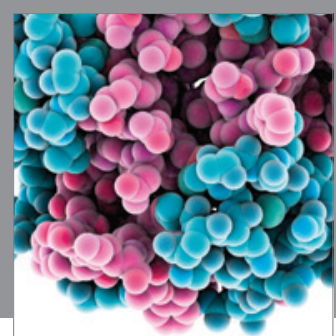

Journal of
Diabetes Research

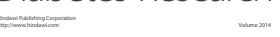

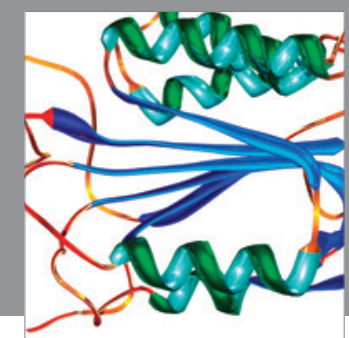

Disease Markers
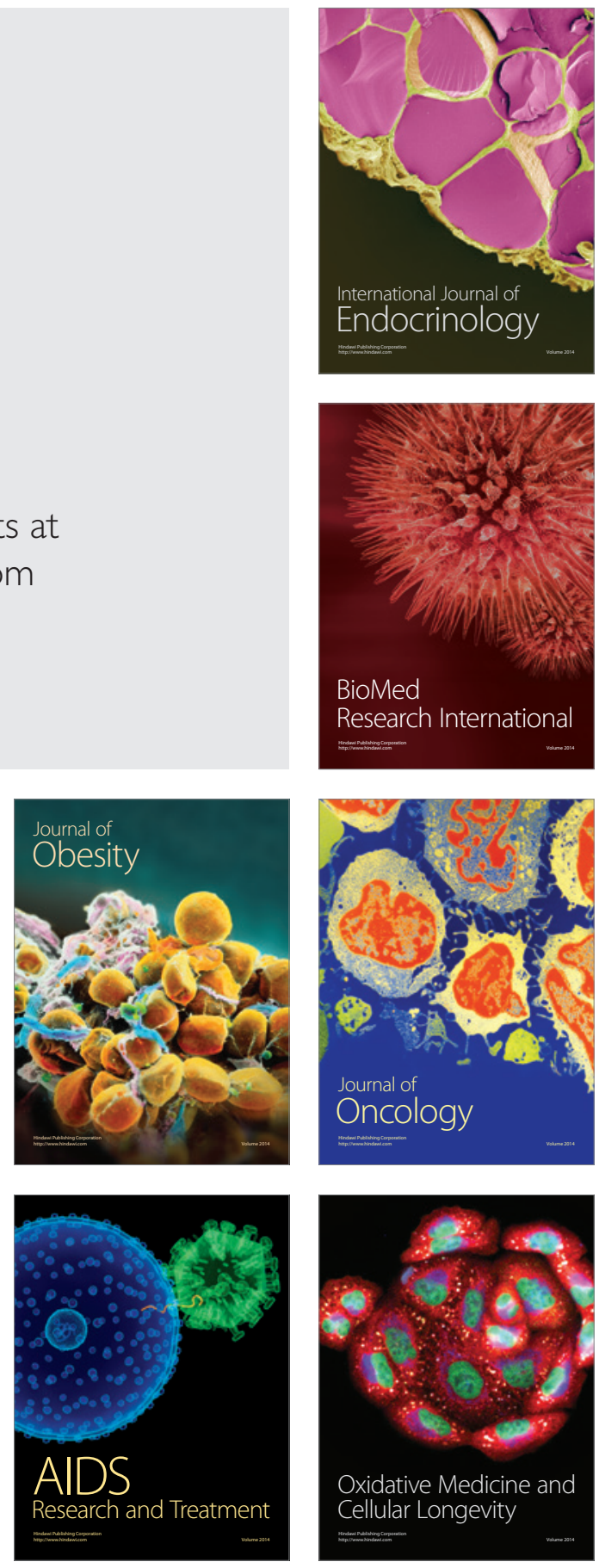